\begin{tabular}{|c|c|}
\hline $\begin{array}{l}\text { LA GRANJA: } \\
\text { REVISTA DE }\end{array}$ & Reseña bibliográfica / Review \\
\hline CIENCIAS DE LA VIDA & Química PREBiótica \\
\hline pISSN:1390-3799; eISSN:1390-8596 & \\
\hline tp://doi.org/10.17163/lgr.n26.2017.01 & 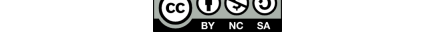 \\
\hline
\end{tabular}

LA GRANJA:

REVISTA DE

ISSN:1390-3799; eISSN:1390-8596

http:/ /doi.org/10.17163/lgr.n26.2017.01
Reseña bibliográfica / Review

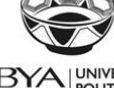

ABYA UNIVERIDAD

YALA POLITECNICA

\title{
LA HISTIDINA COMO UN POSIBLE PRECURSOR EN EL ORIGEN DE LA VIDA
}

\author{
HISTIDINE AS AN ORIGIN OF LIFE POSSIBLE PRECURSOR
}

\author{
Karina Cervantes de la Cruz ${ }^{1,2}$, Isabel Mejía-Luna ${ }^{1}$, \\ Donovan Omar Villanueva Pineda ${ }^{1,2}$, María Colín-García ${ }^{3}$, \\ Alejandro Heredia ${ }^{2, *}$
}

\author{
${ }^{1}$ Departamento de Física, Facultad de Ciencias, UNAM Circuito Exterior s/n, Coyoacán, Universidad Nacional Autónoma de \\ México, Ciudad Universitaria, Cd. Universitaria, 04510 Ciudad de México. \\ 2 Instituto de Ciencias Nucleares, UNAM. Circuito Exterior S/N. Coyoacán, Universidad Nacional Autónoma de México, Cd. \\ Universitaria, 04510 Ciudad de México, D.F. Ciudad de México. \\ 3 Instituto de Geología, UNAM. Circuito de la Investigación Científica, Universidad Nacional Autónoma de México, Ciudad \\ Universitaria, 04360 Ciudad de México, D.F.
}

*Autor para correspondencia: aheredia@nucleares.unam.mx

Manuscrito enviado el 17 de enero de 2017. Aceptado, tras revisión, el 27 de abril de 2017. Publicado el 1 de septiembre de 2017.

\section{Resumen}

Los procesos químicos que se dieron durante los primeros años de la evolución del planeta Tierra, -antes de la presencia de formas celulares-, han sido motivo continuo de estudios a nivel experimental en muchos laboratorios. Considerando ambientes prebióticos plausibles, se ha dado importancia y validez a la presencia de materiales, tales como minerales y arcillas, que pudieron aportar elementos químicos necesarios para catalizar reacciones químicas y estabilizar otro tipo de compuestos orgánicos. La estructura cristalina de algunas biomoléculas de importancia biológica, así como su estereoquímica pueden llevarnos a comprender algunas de las formas de compuestos descritos en el espacio; en particular los compuestos orgánicos mencionados en algunas meteoritas. Finalmente, aportar elementos que nos ayuden a dar nuevas evidencias sobre el ¿cómo? y el ¿por qué? de la existencia de algunas moléculas de importancia biológica, siempre enriquecen el campo científico, y en particular abren nuevos horizontes para entender la relevancia en los procesos fisicoquímicos y más tardíamente, los procesos metabólicos, que pudieron dar lugar a organismos vivos de tipo unicelular en la Tierra primitiva. En el presente trabajo se discute la importancia de la histidina como catalizador orgánico en los estudios sobre el origen de la vida. Se presentan los resultados preliminares sobre la formación de monocristales de histidina en una disolución acuosa y sus posibles implicaciones como aminoácido esencial para la formación de oligopéptidos. Además se plantea la posibilidad de que este aminoácido haya actuado en un momento dado, como catalizador de ciertas reacciones químicas vitales en muchos seres vivos, apoyando su potencial actividad como catalizador orgánico.

Palabras claves: histidina, química prebiótica, complejidad molecular, catalizador. 


\begin{abstract}
The chemical processes occurred during the first years of the evolution of the planet Earth, before the presence of cellular forms, have been continuous reason of studies at experimental level in many laboratories. Considering some possible prebiotic environments, the presence of materials such as clays-type minerals, which could provide chemical and structural elements such as their surfaces, have been given importance and validity to give protection and reactivity to the organic molecules existing in the surrounding environment. These catalytic processes, mediated by mineral surfaces, could give rise in the interstellar medium to a whole range of organic molecules. Many of these are low molecular weights, such as amino acids and carboxylic acids and sometimes molecular weights are much higher. Providing elements that help us to give new evidence about the origin of some molecules of biological importance in the interstellar medium, always enrich the scientific field related to the origin of life, and in particular open new horizons to understand the relevance of physicochemical processes that Could give rise to living organisms on primitive Earth. The present work discusses the possible abiotic synthesis of the amino acid histidine and its importance as an organic catalyst in the formation of oligopeptides in simulations of reactions at the origin of life. In this paper, we discuss the relevance of having histidine monocrystals, simulating a process of hydration-dehydration in shallow pools on the primitive Earth; A phenomenon that is essential for the formation of oligopeptides and, in turn, generate supramolecular assemblies before the appearance of life on our planet.
\end{abstract}

Keywords: histidine, prebiotic chemistry, molecular evolution, molecular complexity, catalyst.

Forma sugerida de citar: Cervantes K., Mejía-Luna I., Villanueva D., Colín-García M., Heredia A. 2017. La histidina como un posible precursor en el origen de la vida. La Granja: Revista de Ciencias de la Vida. Vol. 26(2):6-14. pISSN:1390-3799; eISSN:1390-8596. 


\section{Introducción}

Una de las grandes preguntas de todos los tiempos se centra en cómo se pudo formar la vida y dar lugar a la gran biodiversidad que hoy presenta el planeta Tierra. Más allá de entender el concepto vida, que en muchos momentos puede resultar abstracto por definición, se busca esclarecer cuáles pudieron ser los mecanismos que favorecieron en la Tierra primitiva la síntesis prebiótica, que finalmente dio origen a la vida. Experiencias en laboratorios, partiendo de reacciones con compuestos inorgánicos como el ácido cianhídrico, amoníaco, agua, y dióxido de carbono, entre otros, más la adición de una fuente de energía (p.e. electricidad, luz ultravioleta, radiaciones, etc.), han dado lugar a la formación de ciertas biomoléculas del tipo de aminoácidos, azúcares o moléculas.

\section{De simples moléculas a memoria genética}

Las reacciones de polimerización de compuestos orgánicos con relevancia biológica y de bajo peso molecular (no mayor a $10 \mathrm{kDa}$ ), siempre han mantenido con la lupa en la mano a los científicos para encontrar respuestas sobre la vía que sigue una síntesis y qué productos se forman. Con esta información se pueden equiparar estas reacciones con aquellas presentes en los sistemas vivos. En la década de los ochenta, el investigador Stanley Miller (1986), supuso que algunas de las síntesis prebióticas de pequeñas moléculas inorgánicas se llevaron a cabo en el cuerpo parental de ciertas meteoritas del tipo condritas carbonosas. Estos cuerpos pudieron servir como sitios de reacción para la formación de algunas moléculas relevantes en biología.

Las bases nitrogenadas que identificamos en el DNA, RNA y algunas ribozimas, son las mismas en todos los seres vivos presentes en el planeta en la actualidad; esto es una evidencia apoyada por los avances en genética molecular, bioquímica y filogenia, que apoya la idea del origen común de todos los seres vivos. Más allá de dicha aseveración, la complejidad y capacidad de autorreplicarse y conservar una memoria química sea quizá, el elemento clave para entender, con los componentes biológicos actuales, los mecanismos que dieron origen a la vida. Esta idea no es nueva. Charles Darwin mencionaba en su obra "El origen de las especies" que...
La analogía me llevaría a dar un paso más, o sea a creer que todos los animales y plantas descienden de un solo prototipo; pero la analogía puede ser una guía engañosa. Sin embargo, todos los seres vivientes tienen mucho de común en su composición química, su estructura celular, sus leyes de crecimiento y en ser susceptibles a las influencias nocivas (Cap. XIV Recapitulación y conclusión, 1959).

Estas características determinadas mediante la observación, implican que todos descendemos de un ancestro común hipotético, al cual se ha denominado LUCA (por sus siglas en ingles de Last Universal Common Ancestor). Este organismo, debió contar con la capacidad de autorreproducirse utilizando una maquinaria metabólica almacenando información mediante un código químico, que posteriormente sería interpretado como el código genético. Siendo así, la química brinda una oportunidad para clarificar cómo se formaron los compuestos orgánicos que, luego de muchos procesos, integraron a este posible protobionte o célula primitiva (Mosqueira, Negron-Mendoza, y Ramos-Bernal, 2015).

Las moléculas orgánicas que componen a todos los seres vivos se estructuran principalmente por cadenas de carbono que se encuentran concatenadas por enlaces covalentes. Sin embargo, la presencia de ciertos grupos químicos, como el hidroxilo, amino y carboxilo influyen directamente sobre su arreglo tridimensional, la reactividad química y la estabilidad de las macromoléculas. La distribución espacial de cada molécula determina la funcionalidad y actividad sobre un sustrato o complejo. Los aminoácidos, por ejemplo, cuando se enlazan, constituyen a las proteínas, enormes moléculas que realizan distintas funciones que van desde ser estructurales, enzimáticas, hormonales o transportadoras de oxígeno como el caso particular de la hemoglobina.

Los aminoácidos por su composición química pueden actuar como moléculas cargadas o neutras bajo determinadas condiciones de $\mathrm{pH}$ y temperatura, provocando una reorientación de sus cargas. Algunos aminoácidos forman cristales cuando son expuestos a cambios de temperatura, cuando se incrementa su concentración en soluciones acuosas, si se modifica el $\mathrm{pH}$, o si existe la presencia de otras sales, campos magnéticos, que pueden activar acciones de tipo catalizador al promover la oligomerización entre otros aminoácidos (Mosqueira, NegronMendoza, y Ramos-Bernal, 2015; Sugahara y Mimura, 2014). 
Por ejemplo, la glicina cristalina, obtenida en ciclos de hidratación-deshidratación, promueve reacciones, que aumentan el peso molecular de los otros aminoácidos (Rodríguez-García et al., 2015) y la orientación y elongación de otros enlaces covalentes que modifican la geometría de las fuerzas electrostáticas que forman al nuevo péptido.

\subsection{La importancia de la histidina en el ori- gen de la vida}

La histidina, por su capacidad catalítica y de autoensamblaje (se le llama autoensamblaje a un proceso de unión espontánea de las moléculas, en el que se genera un orden y propiedades supramoleculares) desempeña un papel clave en la evolución química y la química prebiótica. La histidina adquie-

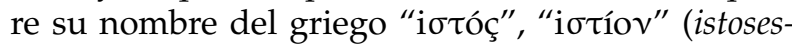
tion) que significa mástil y telar o tejido (Wikipedia, 2016). Es un aminoácido esencial que se caracteriza por la presencia del grupo imidazol (la parte resonante en la Fig. 1) que le confiere propiedades básicas a ella y a sus derivados. Sólo las plantas y algunos microorganismos son capaces de sintetizarla (Coordinación de Enseñanza Bioquímica, Facultad de Medicina UNAM, 2017). Se trata de un aminoácido vinculado a funciones catalíticas, en otras palabras, la histidina está presente en el sitio activo de varias enzimas asociado a la transferencia de electrones. La histidina no solo tiene versatilidad estructural y catalítica, sino que también posee versatilidad metabólica, al ser precursora de los derivados de los aminoácidos metilados como alanina, lisina, metionina, la asparagina, el ácido aspártico y la arginina.

La vida surgió en el Arqueano (o Arcáico) temprano, posterior al último bombardeo intenso (Gómez-Caballero, y Pantoja-Alor, 2003) aproximadamente hace 3.7 millones de años (Nutman et al., 2016). Esto quiere decir que para el momento en que se originó la primera población de células, todos los aminoácidos ya debieron estar formados y acoplados en una maquinaria autorreplicante. Digamos que, en el estudio del origen de la vida, la discusión central es cómo se formaron los compuestos orgánicos primitivos y su posterior organización en la primera población celular (Lazcano-Araujo, 1989 https://www.youtube. $\mathrm{com} /$ watch? $\mathrm{v}=$ Ewad09KhUKc).

Los iones inorgánicos, entre ellos el calcio, los fosfatos o los silicatos, presentes en algunos minera- les, conjuntamente con la histidina, pudieron ayudar a formar moléculas de mayor peso molecular (Plankensteiner, Reiner, y Rode, 2005). Es importante reconocer que las concentraciones de los compuestos orgánicos en la Tierra primitiva pudieron ser bajas (Lazcano-Araujo, 1989), haciéndose necesaria una forma de aumentar su concentración en el lugar donde se lleva la reacción química (Fig. 2)

En el laboratorio de Química de Radiaciones y Radioquímica adscrito al Departamento de Evolución química en el Instituto de Ciencias Nucleares de la Universidad Nacional Autónoma de México, se realizan pruebas de estabilidad de compuestos orgánicos como bases nitrogenadas, ácido carboxÃlicos y aminoácidos principalmente, para corroborar el comportamiento químico y la capacidad de protección que presentan ante la exposición a radiaciones ionizantes bajo condiciones de saturación salina en presencia de arcillas y silicatos. De estudios preliminares obtenidos hasta el momento, se ha podido comprobar que una vez que estos compuestos orgánicos están en contacto con la red cristalina de una arcilla como la montmorillonita de sodio, se pueden cambiar algunas propiedades fisicoquímicas del compuesto orgánico, tal como en el caso de la guanina que aumenta su estabilidad al ser expuesta a radiación gamma (MeléndezLópez, Ramos-Bernal, y Ramírez-Vázquez, 2014) y paralelamente, aumenta la concentración local promoviendo reacciones químicas. Al mismo tiempo, los procesos comunes en ambientes naturales $\mathrm{CO}^{-}$ mo hidratación-deshidratación son capaces de favorecer reacciones de polimerización, provocando un aumento de las masas moleculares de los compuestos presentes. Es decir, la evolución química y la química prebiótica están fuertemente asociadas con aspectos del medio físico como la presencia de agua y de ciertas fases minerales.

\section{Histidina y su posible síntesis pre- biótica}

En condiciones prebióticas, es posible obtener precursores químicos de la histidina y lograr la síntesis de toda la molécula (Shen, et al., 1990) Fig. 3. El paso crítico es la obtención del grupo imidazol, a partir de un aldehído, glioxal y amoníaco. El grupo imidazol es la pieza clave en el funcionamiento de la histidina como catalizador. El formaldehído y el amoníaco son compuestos orgánicos encontrados fácil- 


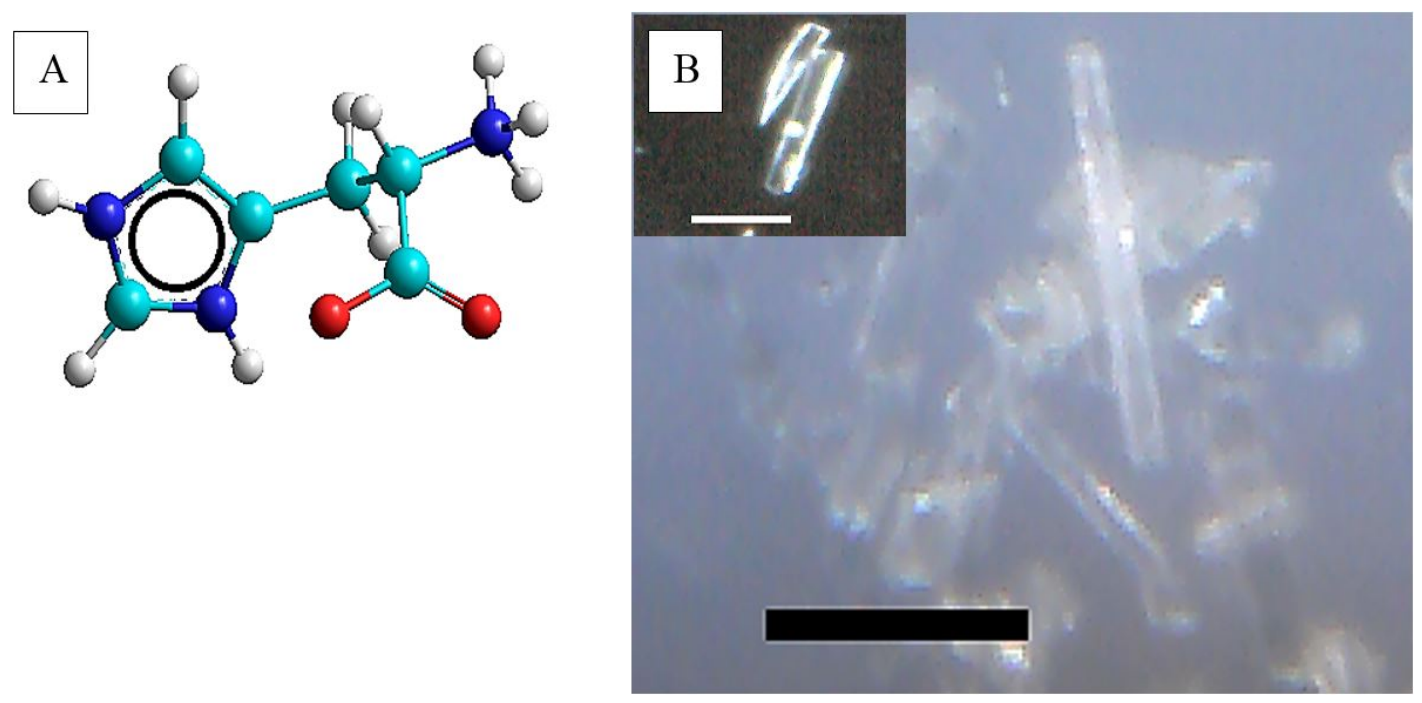

Figura 1. A. Estructura de la histidina, esta posee un grupo imidazol, el anillo que contiene un círculo, representando la resonancia. B. Cristal de histidina obtenido en el laboratorio (barras de escala ca. $100 \mathrm{um}$ ) puede servir como catalizador

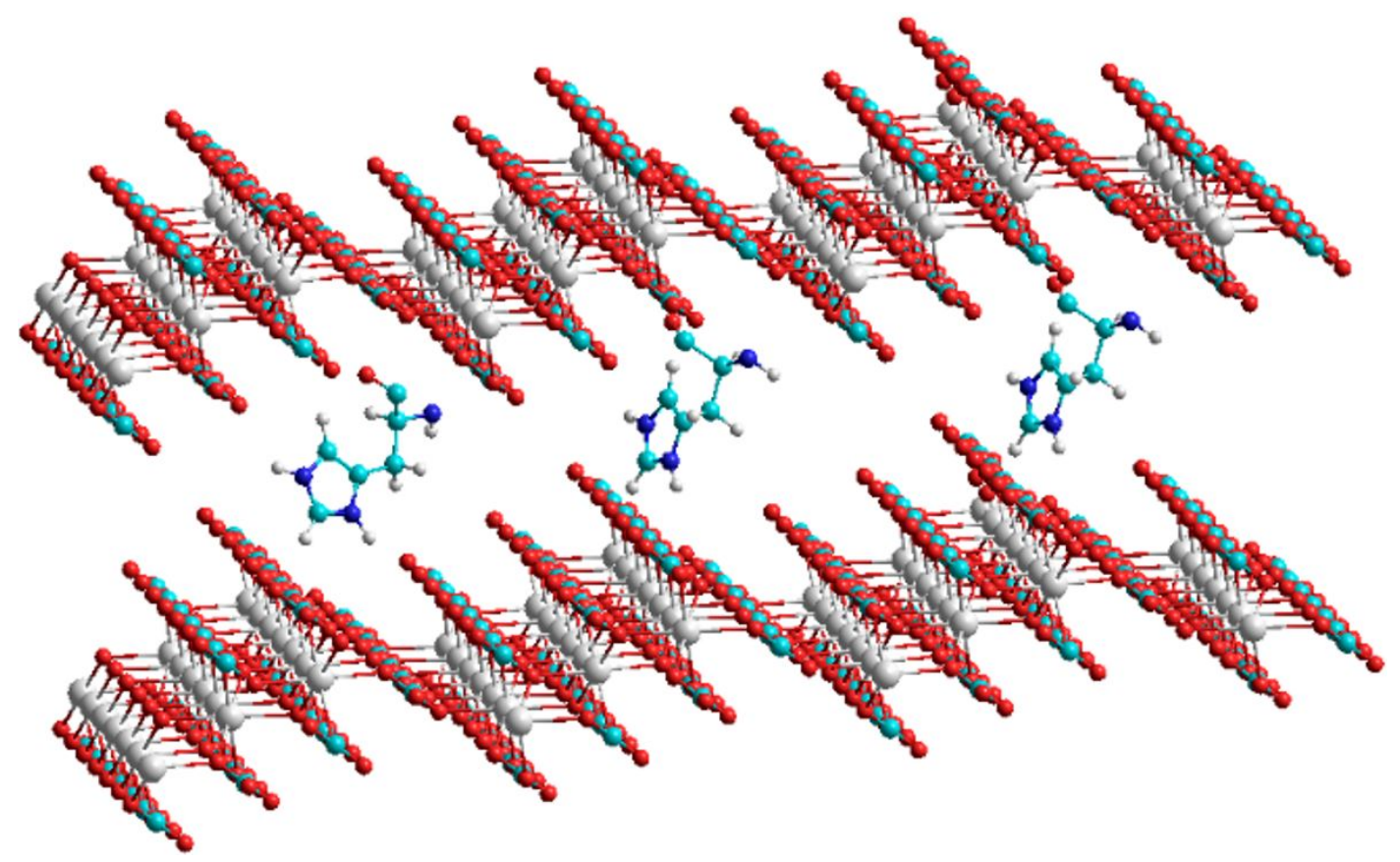

Figura 2. Las superficies minerales pudieron ser un centro de concentración de compuestos orgánicos. En estos, las interacciones electrostáticas y las del tipo puente de hidrógeno pudieron ser de gran relevancia. En la figura se muestra la interacción entre moléculas de histidina y una montmorillonita simulada computacionalmente. 
mente en el universo, específicamente en nubes moleculares y meteoritas (Miller, 1986; Ehrenfreund, y Charnley, 2000). Si la síntesis de estos compuestos se dio espontáneamente en el universo, entonces, se puede pensar que la Tierra primitiva también tuvo este tipo de compuestos que a la postre pudieron formar estas biomoléculas de forma abiótica.

En la formación de aminoácidos algunas reacciones son clave, una de estas reacciones es la síntesis de Strecker. El imidazol-4-formaldehído se convierte a histidina a través de esta reacción (Fig. 4).

La reacción de Strecker (1850) es en realidad un conjunto de reacciones químicas, que generan aminoácidos a partir de aldehídos o cetonas. Adicionalmente, la histidina, pudo coadyuvar a la formación de otros compuestos de gran relevancia biológica como las llamadas "monedas energéticas" (Shen, et al., 1990, 2016) funcionando como catalizador. En los organismos actuales, la formación de estas "monedas" está mediada por la histidina. Ello nos hace suponer que la histidina pudo generar reacciones químicas parecidas a las que vemos en el laboratorio actualmente, por lo que su síntesis fue crucial para la química prebiótica.

\section{El grupo imidazol viajando en el medio interestelar}

No se tiene mucha información de la presencia de histidina en cuerpos extraterrestres. Por otro lado, la presencia del grupo imidazol se ha confirmado en distintas meteoritas carbonosas (Ehrenfreund, y Charnley, 2000) como el Murchison (Oba, y Narao$\mathrm{ka}, 2006)$. Esto nos hace suponer que la síntesis de este compuesto pudo darse durante su viaje espacial. Una alternativa, es que la química de impactos podría ofrecer caminos adicionales para que se sintetizara la molécula en su caída a la Tierra, aunque otras posibilidades existen, como la de ser formada por reacciones de superficie sobre los minerales irradiados por las emisiones de un Sol joven (Gaustad, y Vogel, 1982). Por otro lado, las moléculas complejas halladas en el meteorito Murchison son ya moléculas complejas (Fig. 3 y 4). En nuestros estudios, consideramos importante simular computacionalmente los ambientes fisicoquímicos moleculares para formar moléculas del tipo de la histidina, que como se menciona anteriormente, no se ha encontrado $m \tilde{A}_{j}$ s que en la Tierra, como producto de actividad biológica. Usando este tipo de herra- mientas se podría determinar a qué presiones, temperaturas y cuál de los derivados del imidazol, podrían reaccionar con mayor facilidad para dar origen a este importante aminoácido.

La evolución molecular es un paso más en la complicación de la materia orgánica. Por ejemplo, se puede decir que una molécula lineal es de menor complejidad a una molécula ramificada, aunque tenga su misma masa molecular. Esto es un ejemplo de evolución molecular (Fig. 6).

En el caso de los aminoácidos, un aumento en masa molecular puede conducir a la formación de péptidos, y una vez más, la química de impactos, podría ser promotora de estas reacciones de polimerización. En este panorama de evolución química para formar al aminoácido histidina, tenemos algunas observaciones relevantes que considerar. Por ejemplo, los precursores de la histidina y los de las bases nucleotídicas son semejantes. El hecho es que la histidina se comporta químicamente de forma muy versátil, lo que podría traer respuestas a las rutas de síntesis de ambos grupos de compuestos. En estos casos los modelos computacionales son necesarios para obtener datos adicionales al fenómeno estudiado.

\section{Conclusiones}

Aquí revisamos el panorama actual de los estudios que vinculan la estructura de la histidina y su capacidad química de tener distintas propiedades fisicoquícas. Estos datos nos ayudan a entender que esta molécula, relativamente simple, pudo tener un papel relevante en la transformación química local antes de la aparición de la vida. Actualmente, nuestro interés va encaminado a investigar las consecuencias de la interacción de la histidina e imidazol con otros aminoácidos, con iones inorgánicos y minerales ricos en fosfatos. En nuestros resultados preliminares, conseguimos formar cristales de histidina y a estos mismos se les va a someter a experimentos de irradiación con radiación gamma y a procesos de hidratación-deshidratación, que nos llevarán a entender la ruta molecular por la cual la histidina se comporta como catalizador. Queremos entender cómo estos compuestos químicos lograron transformarse en otras biomoléculas de mayores pesos moleculares y si podemos situar esta transformación química en un fenómeno puntual que coadyuve a conocer más sobre la historia prebiótica en la Tierra 

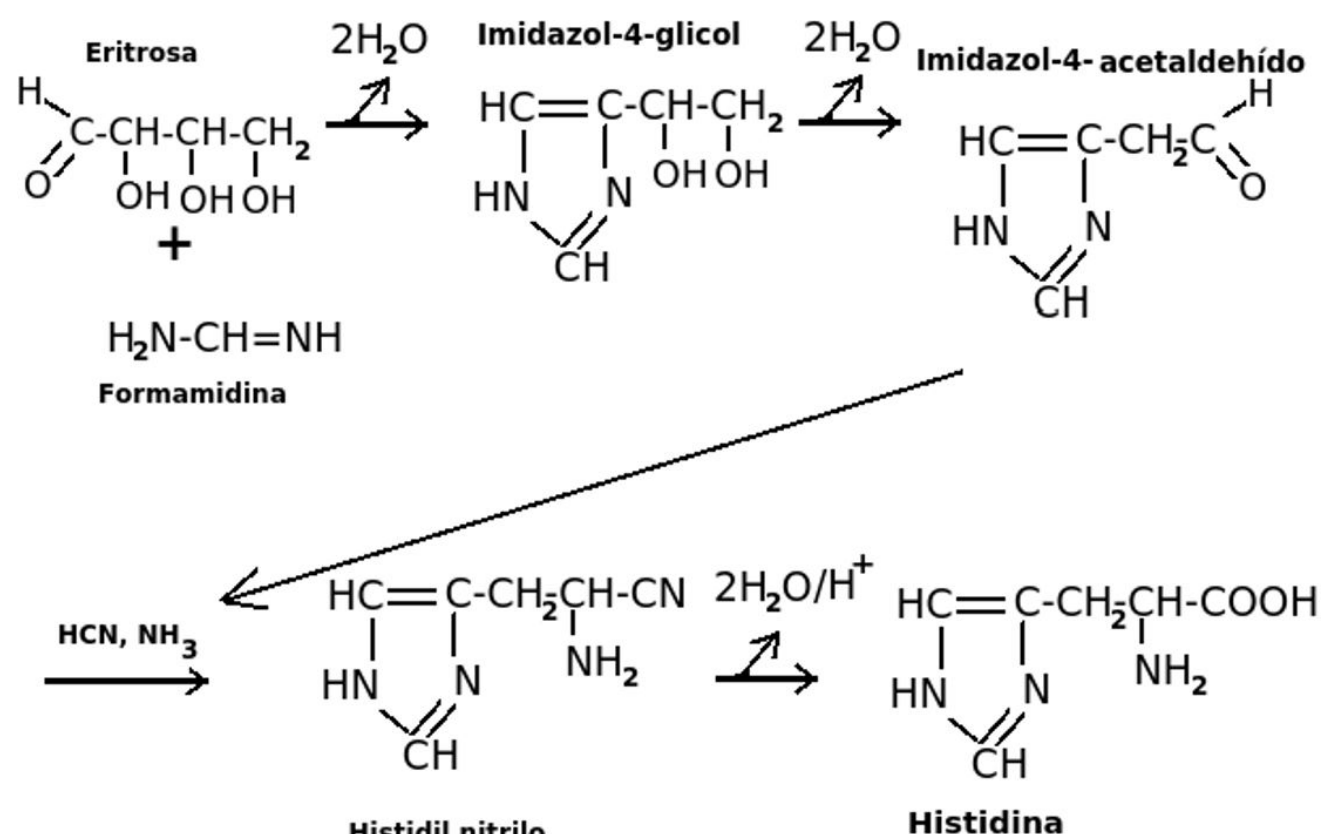

Figura 3. Formación de histidina a partir de eritrosa (un azúcar). Se puede observar que la síntesis de este aminoácido implica la pérdida de varias moléculas de agua, para lograr la formación del anillo de imidazol.

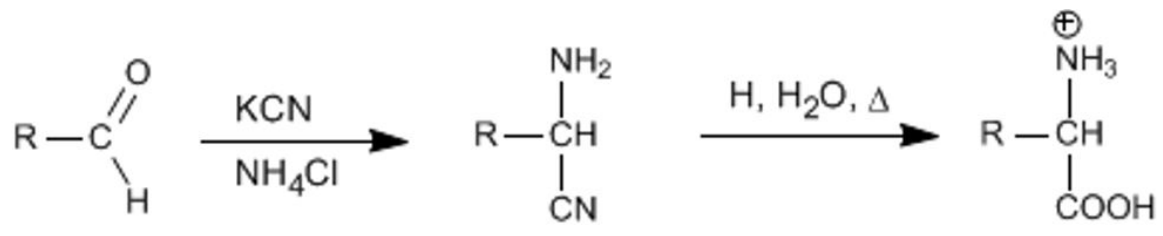

Figura 4. Mediante la reacción de Strecker se sintetizan aminoácidos de forma abiótica.

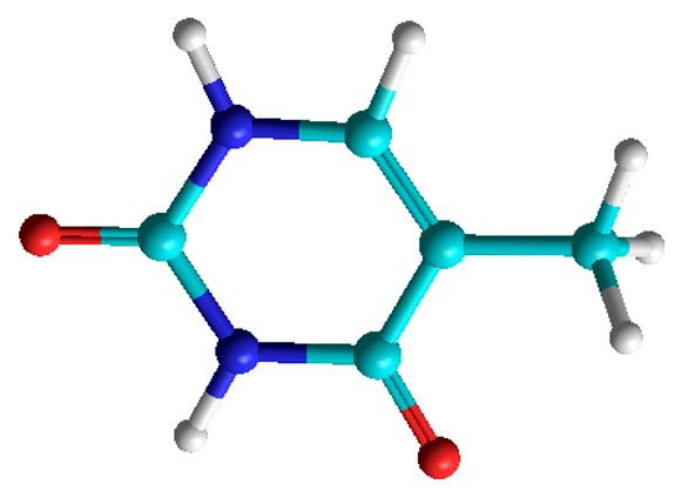

Figura 5. La timina, se encontró en el meteorito Murchison. 

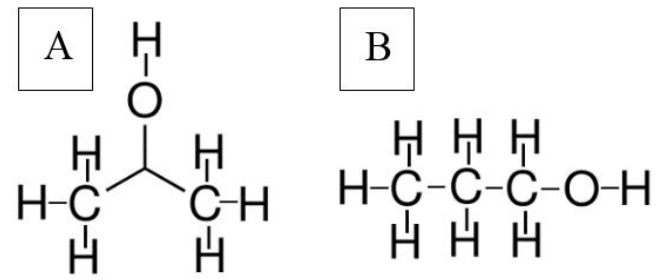

Figura 6. Una misma masa molecular puede dar origen a varias geometrías y esto es un ejemplo de evolución molecular. El isopropanol (A) y el propanol (B) son un ejemplo de variación geométrica de la molécula sin variar la masa molecular (masa molecular de ambos es de $60 \mathrm{~g} / \mathrm{mol})$.

y el universo. En general, nuestros proyectos quieren transferir datos de evolución química a fenómenos de evolución molecular para explicar en la medida de lo posible el origen de la vida.

\section{Agradecimientos}

Agradecemos a Luciano Díaz González, Martín Cruz Villafañe, Luis Miguel Valdez Pérez, Ing. Juan Eduardo Murrieta León y Mat. Enrique Palacios Boneta por su asistencia en las simulaciones computacionales y al Mtro. Benjamín Leal Acevedo, Téc. Académ. Francisco García Flores y Aux. de Laboratorio Francisco Isidoro Pacheco por la asistencia en irradiación de muestras.

\section{Referencias}

Coordinación de enseñanza bioquímica facultad de medicina, UNAM. Imágenes del libro Lehninger [En línea] Disponible en: https://goo.gl/ q8wh4z. (Consultado: 16-enero-2017)

Darwin, Ch..El origen de las especies. [En línea] Disponible en: https:/ / goo.gl/YLhupZ. (Consultado: 11-enero-2017)

Ehrenfreund, P. y Charnley, S. B., 2000. Organic Molecules in the Interstellar Medium, Comets, and Meteorites: A Voyage from Dark Clouds to the Early Earth. Annual Review of Astronomy and Astrophysics, 38(1), pp. 427-483. DOI: https://doi.org/10.1146/annurev.astro. 38.1.427

Gaustad J. E. y Vogel S. N., 1982. High energy solar radiation and the origin of life. Origins of Life and Evolution of Biospheres, 12(1), pp. 3-8. DOI: https://doi.org/10.1007/BF00926907
Gómez-Caballero, A. y Pantoja-Alor, J., 2003. El origen de la vida desde un punto de vista geológico. Boletín de la Sociedad Geoógica Mexícana Tomo LVI, 1, pp. 56-86. Disponible en: https://goo.gl/cCXoR7

Lazcano-Araujo, A., 1989. El origen de la vida: evolución química y evolución biológica, México: Trillas. Disponible en: https:/ /goo.gl/Be3oT8

Meléndez-López, A. L., Ramos-Bernal S., Ramírez-Vázquez, M. L., 2014. Stability of guanine adsorbed in a clay mineral under gamma irradiation at temperatures (77 and $298 \mathrm{~K})$ : Implications for chemical evolution studies. AIP Conference Proceedings, 1607(1), pp. 111-115, DOI: http://dx.doi.org/10.1063/ 1.4890710

Miller, S. L., 1986. Current status of the prebiotic synthesis of small molecules. Chemica Scripta, 26B, pp. 5-11. Disponible en: https://goo.gl/ YBNuQs

Mosqueira, F. G., Negrón-Mendoza, A., RamosBernal, S., 2015. An interpretation of the oligomerization of amino acids under prebiotic conditions Boletín de la Sociedad Geoógica Mexícana, 67(3), pp. 421-432. Disponible en: https: //goo.gl/9x5QJX

Nutman, A. P., Bennett, V. C., Friend, C. R. L., Van Kranendonk, M. J., Chivas, A. R., 2016. Rapid emergence of life shown by discovery of 3,700-million-year-old microbial structures. Nature, 537(7621), pp. 535-538. Disponible en: https://goo.gl/PZRNi3

Oba, Y., y Naraoka, H., 2006. Carbon isotopic composition of acetic acid generated 
by hydrous pyrolysis of macromolecular organic matter from the Murchison meteorite. Meteoritics \& Planetary Science, 41(8), pp. 1175-1181. DOI: https://doi.org/10.1111/ j.1945-5100.2006.tb00514.x

Plankensteiner, K., Reiner, H. y Rode, B. M., 2005. Stereoselective differentiation in the Salt-induced Peptide Formation reaction and its relevance for the origin of life. Peptides, 26(4), pp. 535-541. DOI: https://doi.org/10. 1016/j.peptides.2004.11.019

Rodríguez-García, M., Surman, A. J., Cooper, G. J., Suárez-Marina, I., Hosni, Z., Lee, M. P., y Cronin, L., 2015. Formation of oligopeptides in high yield under simple programmable conditions. Nature communications, 6(8385), pp. 1-7. DOI: https://doi.org/

\subsection{8/ncomms9385}

Shen, C., Yang, L., Miller, S. L. y Oró, J., 1990. Prebiotic synthesis of histidine. Journal of Molecular Evolution, 31(3), pp. 167-174. DOI: https: //doi.org/10.1007/BF02109492

Shen, C., Yang, L., Miller, S. L. y Oró, J., 1986. Prebiotic synthesis of imidazole-4-acetaldehyde and histidine. Origins of life and evolution of the biosphere, 17(3-4), pp. 295-305. DOI: https: //doi.org/10.1007/BF02386469

Sugahara, H. y Mimura, K., 2014. Glycine oligomerization up to triglycine by shock experiments simulating comet impacts. Geochemical Journal, 48(1), pp. 51-62. DOI: http://doi.org/ 10.2343/geochemj.2.0285

Wikipedia, 2016. 